\title{
Combined accuracy of optic nerve sheath diameter, strain ratio, and shear wave elastography of the optic nerve in patients with idiopathic intracranial hypertension
}

\author{
Ahmed Abdel Khalek Abdel Razek', Nada Elsaid², Tamer Belal', Nihal Batouty', \\ Ahmed Azab²
}

Departments of ${ }^{1}$ Diagnostic Radiology and ${ }^{2}$ Neurology, Mansoura Faculty of Medicine, Mansoura, Egypt

Purpose: This study assessed the combined accuracy of optic nerve sheath diameter (ONSD), strain ratio (SR), and shear wave elastography (SWE) of the optic nerve (ON) in patients with idiopathic intracranial hypertension (IIH).

Methods: This prospective study was carried out on both ONs of 34 consecutive patients diagnosed with $\mathrm{IH}$ and 16 age- and sex-matched healthy volunteers. All of the study participants were women. The ONSD, SR, and SWE of the ON were measured. The severity of papilledema of IIH patients was sub-classified into mild papilledema and moderate/severe papilledema.

Results: The mean ONSD $(5.6 \pm 1.1 \mathrm{~mm}), \mathrm{SR}(0.7 \pm 0.1)$, and SWE $(30.1 \pm 16.7 \mathrm{kPa})$ of the IIH patients were significantly different $(P=0.001)$ from the ONSD $(4.1 \pm 0.5 \mathrm{~mm}), S R(0.9 \pm 0.1)$, and SWE $(8.2 \pm 3.4 \mathrm{kPa})$ of controls. The cutoff values of ONSD, SR, and SWE of the ON for differentiating $\mathrm{IH}$ patients from controls were $5.45 \mathrm{~mm}, 0.8$, and $10.3 \mathrm{kPa}$ with areas under the curve (AUCS) of $0.91,0.86$, and 0.96 and accuracy values of $91 \%, 81 \%$, and $93 \%$, respectively. Combined SWE and ONSD and combined SWE, ONSD, and SR for differentiating IIH patients from controls revealed AUCs of 0.98 and 0.99 and accuracy values of $96 \%$ and $96 \%$, respectively. ONSD, SR, and SWE showed significant differences between mild and moderate/ severe papilledema ( $P=0.001)$. Papilledema was correlated with SWE $(r=0.8, P=0.001)$, ONSD $(r=0.4, P=0.001)$, and $S R(r=-0.4, P=0.001)$.

Conclusion: The combination of ONSD, SR, and SWE may be helpful for diagnosing $\mathrm{IH}$, and a good indicator of the degree of papilledema.

Keywords: Idiopathic intracranial hypertension; Optic nerve; Ultrasound elastography

Key points: Optic nerve sheath diameter (ONSD), strain ratio (SR) and shear wave elastography (SWE) are good indicator of papilledema. For diagnosis of idiopathic intracranial hypertension, sonographic combination of ONSD, SR and SWE is helpful.

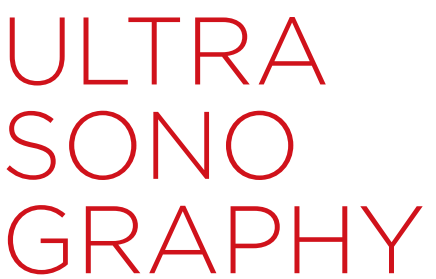

ORIGINAL ARTICLE

https://doi.org/10.14366/usg.20165 pISSN: 2288-5919 e elSSN: 2288-5943 Ultrasonography 2022;41:106-113

Received: October 17, 2020

Revised: April 9, 2021

Accepted: April 20, 2021

Correspondence to:

Ahmed Abdel Khalek Abdel Razek, MD Department of Diagnostic Radiology, Mansoura Faculty of Medicine, Mansoura 13351, Egypt

Tel. +20-161948567

Fax. +20-502315105

E-mail: arazek@mans.edu.eg

This is an Open Access article distributed under the terms of the Creative Commons Attribution NonCommercial License (http://creativecommons.org/ licenses/by-nc/4.0/) which permits unrestricted noncommercial use, distribution, and reproduction in any medium, provided the original work is properly cited.

Copyright @ 2022 Korean Society of Ultrasound in Medicine (KSUM)

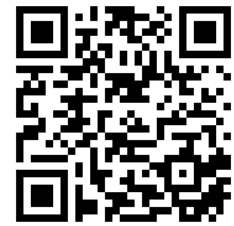

How to cite this article:

Razek AAKA, Elsaid N, Belal T, Batouty N, Azab A. Combined accuracy of optic nerve sheath diameter, strain ratio, and shear wave elastography of the optic nerve in patients with idiopathic intracranial hypertension. Ultrasonography. 2022 Jan;41(1):106-113. 


\section{Introduction}

Idiopathic intracranial hypertension $(\mathrm{IHH})$ is a clinical syndrome of raised intracranial pressure, in the absence of space-occupying lesions, without ventricular enlargement, for which no causative factor can be identified [1-4]. Papilledema and non-specific visual alterations including transient visual obscurations, photophobia, retrobulbar pain, diplopia, and eventually visual loss are the main ophthalmological manifestations of $\mathrm{IH}$. Although visual alterations occur in about $75 \%$ of $\mathrm{IH}$ patients, their mechanism is still poorly understood. It may seem obvious that elevation of the intracranial pressure (ICP) induces papilledema, which in turn causes visual deterioration. However, the pressure-induced effect on the retrobulbar optic nerve (ON) may play a role in visual disturbances [4-7]. There are non-specific findings on routine magnetic resonance (MR) imaging for the diagnosis of IIH. Nonspecific signs of ON involvement are seen on routine MR imaging of IIH patients as a kinked, tortuous ON and ON sheaths with filled-out cerebrospinal fluid (CSF) spaces next to the globe. The role of innovative MR imaging methods such as diffusion tensor imaging, and susceptibility-weighted imaging as biomarkers of ON involvement in IIH is developing [8-14].

The ON sheath diameter (ONSD) measured using ultrasonography is a noninvasive marker of $\mathrm{IH}$. The advantages of ultrasonography include its speed, reduced cost, relative ease of use, repeatability, and lack of side effects [15-22]. Elastography is an imaging modality used with ultrasonography to identify differences in the mechanical elasticity of tissues. Ultrasound elastography analyses tissue elasticity by generating low-frequency vibrations that stimulate strain in tissues, which is subsequently analyzed. Elastography studies the response of the tissues to the action of a force. The stiffness of tissue can be calculated using qualitative or quantitative elastography. Strain elastography (SE) is a qualitative method that measures external compression of the tissues, while shear wave elastography (SWE) is a quantitative method that measures wave velocity generated by a transducer $[23,24]$. Few studies have highlighted the role of elastography of the $\mathrm{ON}$ in detection of the biomechanical ON changes associated with multiple sclerosis, Behçet disease, retrobulbar neuritis, migraine, glaucoma, pre-eclampsia, and Graves disease [23-28]. Only one recent study used SE and SWE to evaluate the ON in patients with IIH [29]. To our knowledge, this is the first study to assess the combined accuracy of these ON parameters in patients with $\mathrm{IIH}$ and the first to evaluate the role of the strain ratio (SR) in the evaluation of the ON.

The aim of this work was to assess the possible role of combined ONSD, SE, and SWE of the ON in the diagnosis of IIH.

\section{Materials and Methods}

\section{Compliance with Ethical Standards}

Mansoura University Faculty of Medicine Institutional review board approval was obtained (IRB approval code: MD. 18.08.74), and informed consent from all patients were obtained.

\section{Study Population}

The inclusion criteria for participation in this study were adult patients with IIH diagnosed according to the modified Dandy diagnostic criteria [30]. The following patients were excluded from our study: patients with a disturbed consciousness level $(n=3)$ and patients that refused to undergo ultrasonography $(n=3)$. Between August 2019 and September 2020, this prospective study was carried out on both ONs of 34 consecutive patients diagnosed with $\mathrm{IIH}$ and 16 age- and sex-matched healthy volunteers with no symptoms or signs suggesting cranial pathology. All of the study participants were women, and the mean age of the patients was $29 \pm 7$ years versus $30 \pm 9$ years for the controls $(P=0.421)$.

\section{Clinical Assessment}

Papilledema grading was done by two neurologists (N.E. and T.B.; 10 and 20 years' experience respectively) who were blinded to patients' clinical presentation and ultrasound findings. The time between the fundus examination and the ultrasound examination was 2 days. The severity of papilledema was classified as mild, moderate, and severe [31]; then, patients were categorized into mild papilledema and moderate/severe papilledema groups, as increased disc edema is positively correlated with increased visual loss [32-34], which in turn influences therapeutic choices [35].

\section{Ultrasonography}

Ultrasonography and elastography were done by a radiologist and another neurologist (N.B. and N.E.; 15 and 10 years' experience respectively), who were blinded to the clinical findings, using an ultrasound machine (Applio 500, Canon Medical Systems Corp., Tokyo, Japan) with a linear array probe of $9 \mathrm{~L}$ and $6 \mathrm{e} 15 \mathrm{~L} \mathrm{MHz}$, and the mean values of their measurements were used for subsequent statistical analyses. The ultrasound examination was performed with the patient supine and eyes closed. A gel was used between the eyelids and the probe to obtain the image. The ONSD on both sides was measured at $3 \mathrm{~mm}$ behind the globe, perpendicular to the long axis of the ON between the external edges of the hyperechogenic area surrounding the ON (Fig. 1A).

\section{Strain Elastography}

The B-mode ultrasonography and elastography results were 
displayed simultaneously on a side-by-side split-screen display. SE images were produced by compression from the transducer. There was no standard number of compressions. Our reference was the SE compression bar to obtain a homogeneous compression wave and optimal filling. Adequate compression was achieved when a yellow color filled the bar. The color diagram of SE ranged from blue to red, where blue represented low stiffness, green represented intermediate stiffness, and red represented high stiffness. The SR was automatically (not manually) calculated depending on the sequence of placement of the regions of interest (ROIs). The first placed ROI was automatically labeled as the target ROI, and the second was automatically labeled as the reference ROI. We were obligated to place the ROls in this sequence as the optic disc was the landmark for placement of the target ROI (first ROI) on the anterior segment of the ON just behind the optic disc, and consequently, the reference ROI (second ROI) was placed posterior to it (Fig. 1B).
Strain ratio

Reference ROI (placed in the posterior segment of the
$=\frac{\text { intraorbital part) }}{\text { Target ROI (placed in the anterior segment of the intraorbital }}$ part)

The SR was calculated from three optimal frames and the mean value was deduced and recorded for further statistical analysis.

\section{Shear Wave Elastography}

The SWE images were produced from static images and were recorded digitally on the machine for subsequent identification. SWE provides a quantitative analysis of the $\mathrm{ON}$ stiffness by measuring the pressure in the elasticity imaging interval $(0-150 \mathrm{kPa})$. Two circular ROls were placed on each $\mathrm{ON}$, and the ROI quantitative values were then assessed using at least three measurements, and the mean values of these measurements were used for subsequent statistical analyses (Fig. 1C).

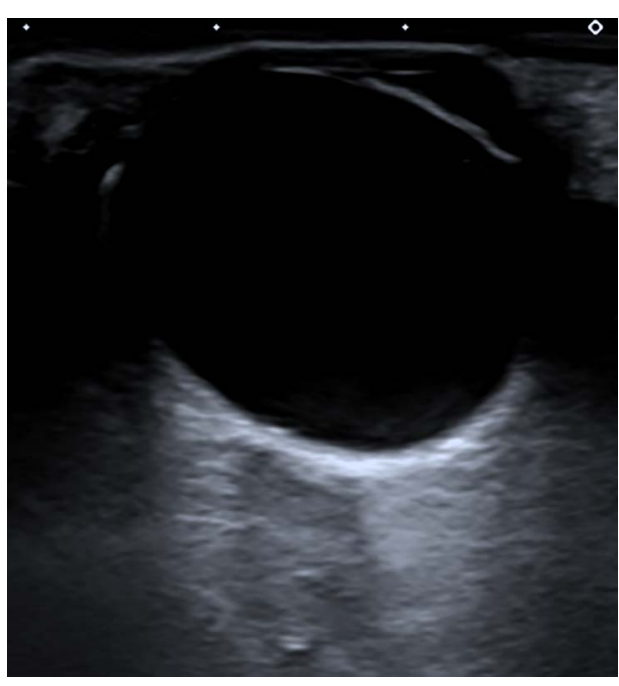

A

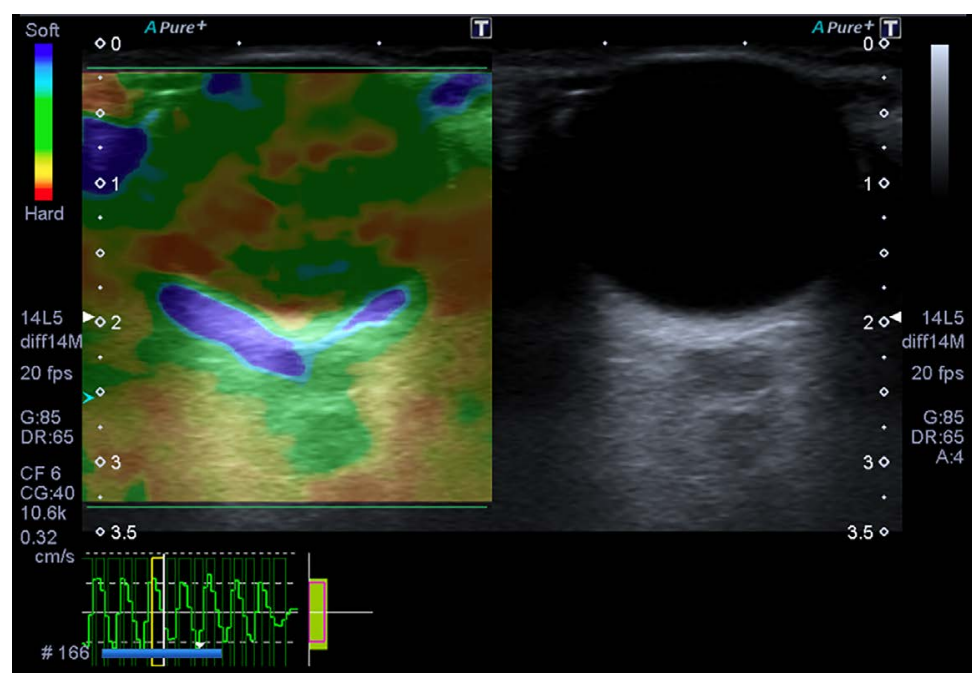

B

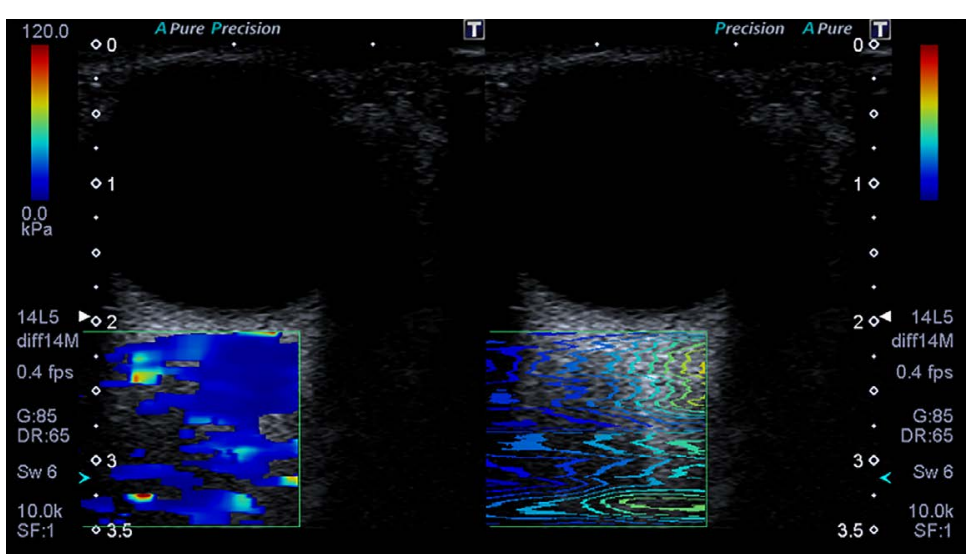

C
Fig. 1. Optic nerve sheath diameter (ONSD), strain ratio $(\mathrm{SR})$, and shear wave elastography (SWE) of the optic nerve (ON) in a normal control subject.

A-C. ONSD measured on ultrasonography was $4.2 \mathrm{~mm}$. (A), strain elastography shows that the SR of the $\mathrm{ON}$ is $0.88(\mathrm{~B})$, and SWE shows that the SWE of the ON is $12.3 \mathrm{kPa}(\mathrm{C})$. 


\section{Statistical Analysis}

Data were analyzed using SPSS version 22.0 (IBM Corp., Armonk, NY, USA). Quantitative data were described as means and standard deviations. The significance of the obtained results was judged at the 0.05 level. After testing normality using the Kolmogorov-Smirnov test, the Student t test was used to compare values between patients and the control group, and between the two groups of patients. Receiver operating characteristic (ROC) curve analysis was used to test the accuracy of SWE, SR, ONSD, and combined parameters to discriminate patients from the control group. The area under the curve (AUC), sensitivity, and specificity were determined from the curve, and the accuracy was calculated through crosstabulation. Multivariate logistic regression analysis for combined SWE, ONSD, and combined SWE, SR, ONSD to discriminate patients from the control group was carried out. Spearman rank-order correlations were used to determine the strength and direction of the linear relationships between papilledema severity and SWE, SR, and ONSD.

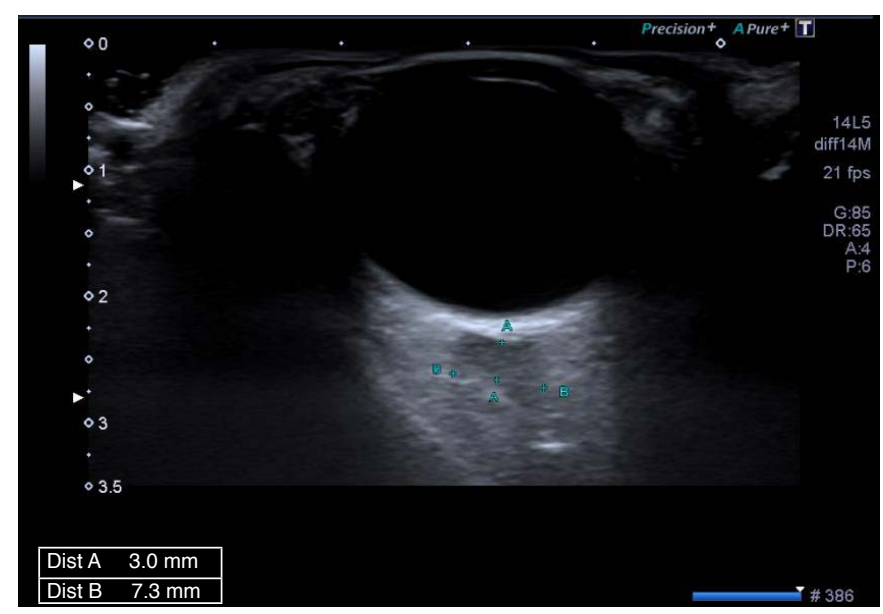

A

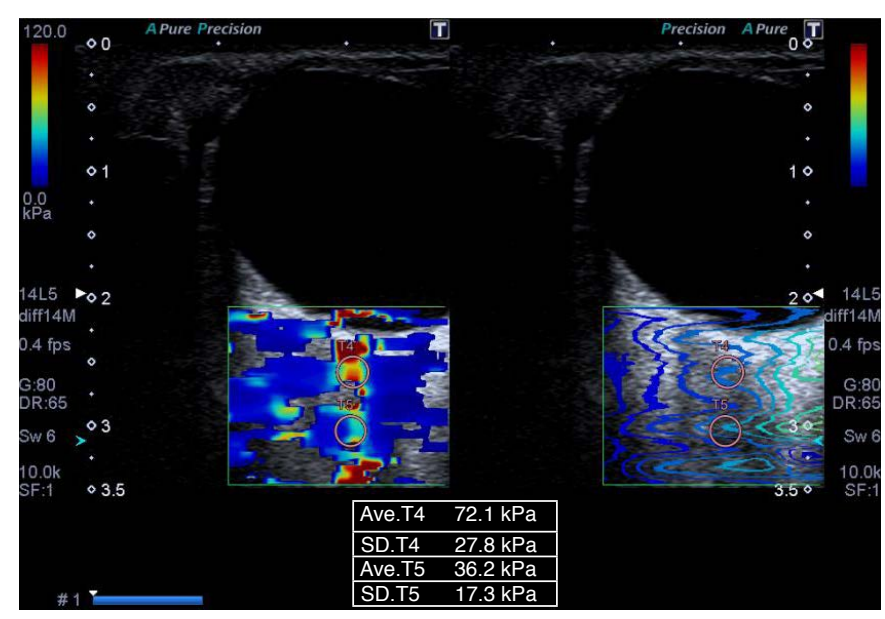

\section{Results}

All patients and controls were women; the mean age of the patients was $29 \pm 7$ years and that of the controls was $30 \pm 9$ years $(P=0.421)$. Fig. 2 presents the degree of papilledema of patients with $\mathrm{IH}$. The mean ONSD $(5.6 \pm 1.1 \mathrm{~mm}), \mathrm{SR}(0.7 \pm 0.1)$, and SWE $(30.1 \pm 16.7 \mathrm{kPa})$ of IIH patients were significantly different $(\mathrm{P}=0.001)$ from the ONSD $(4.1 \pm 0.5 \mathrm{~mm}), \mathrm{SR}(0.9 \pm 0.1)$, and SWE $(8.2 \pm 3.4 \mathrm{kPa})$ of the controls (Table 1). As a cutoff value of ONSD, $5.45 \mathrm{~mm}$ showed the best results (AUC, 0.91; sensitivity, 89\%; specificity, 93\%; and accuracy, $91 \%$ ). The best cutoff value of SR was 0.8 (AUC, 0.86 ; sensitivity, $83 \%$; specificity, $75 \%$; and accuracy, $81 \%$ ). The best cutoff value of SWE (10.3) had an AUC of 0.96 , accuracy of $93 \%$, sensitivity of $98 \%$ and specificity of $81 \%$ (Fig. 3A). According to the data deduced from the ROC curve analysis (Table 2), SR was the least accurate, least sensitive, and least specific parameter. Combining SE and ONSD and combining SWE, SR, and ONSD increased the ability to discriminate patients from the control group (AUC, 0.98 and 0.99;

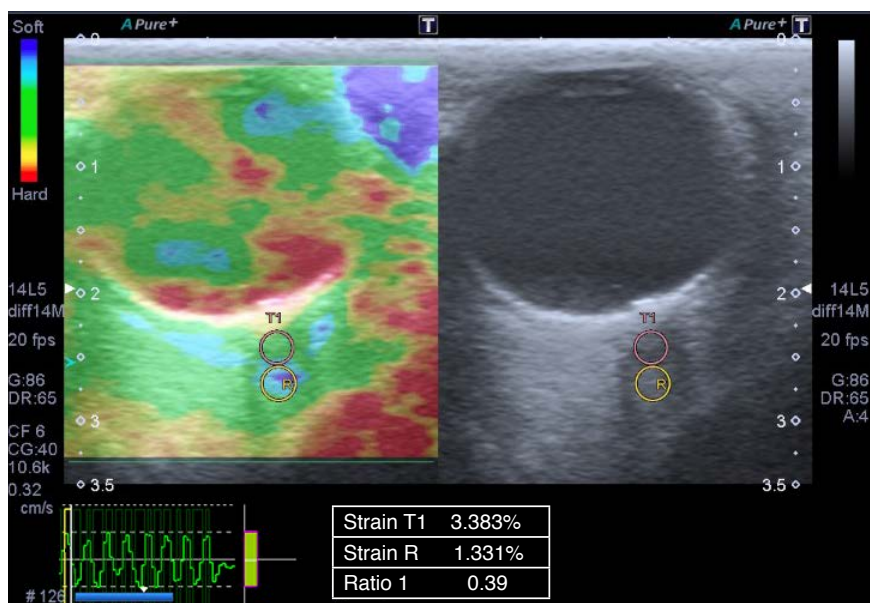

B

Fig. 2. Optic nerve sheath diameter (ONSD), strain ratio (SR), and shear wave elastography (SWE) of the optic nerve (ON) in a patient with idiopathic intracranial hypertension (IIH).

A-C. Ultrasound image shows an ONSD measurement $(7.3 \mathrm{~mm})$ (A), strain elastography shows that the SR of the $\mathrm{ON}$ is $0.39(\mathrm{~B})$, and SWE shows that the SWE of the ON is $36.2 \mathrm{kPa}$ (C). The combined increase in the ONSD, low SR of the ON, and high SWE of the ON helped in the correct diagnosis of $\mathrm{IH}$. 
sensitivity, $98 \%$ and $95 \%$; specificity, $90 \%$ and $96 \%$; and accuracy, $96 \%$ and $96 \%$, respectively) (Table 2, Fig. 3B).

Forty-nine ONs had mild papilledema and 19 had moderate/ severe papilledema. The mean ONSD was $5.4 \pm 0.9 \mathrm{~mm}$, SWE was $23.9 \pm 13.9 \mathrm{kPa}$, and SR was $0.8 \pm 0.2$ in the mild papilledema group. The mean ONSD was $6.4 \pm 0.9 \mathrm{~mm}$, SWE was $46.2 \pm 11.9 \mathrm{kPa}$, and SR was $0.6 \pm 0.1$ in the moderate/severe papilledema group. ONSD, $S R$, and SWE showed significant differences between patients with mild papilledema and those with moderate and severe papilledema ( $P=0.001$ for all) (Table 1). SWE showed a strong positive correlation with papilledema severity $(r=0.8, P=0.001)$. ONSD had a positive correlation with papilledema severity but less than that of SWE ( $r=0.4, P=0.001$ for both). SR was inversely correlated with papilledema severity $(r=-0.4, P=0.001)$.

\section{Discussion}

The main findings of the study are the increased ONSD and decreased $\mathrm{ON}$ elasticity in IIH patients. As a multiparametric indicator, the combination of ONSD, SR, and SWE may help in the diagnosis of $\mathrm{IH}$. These ultrasonographic and elastographic parameters of the $\mathrm{ON}$ were closely correlated with the severity of papilledema, with SWE being the most reliable parameter.

Ultrasonography is commonly used to measure ONSD in the assessment of IIH patients as an easy bedside tool. ONSD is considered an indirect, noninvasive measure of ICP, making it a useful parameter in the clinical diagnosis of IIH [15-17]. The measured ONSD was significantly higher in the IIH group than in the control group ( $P=0.001)$. Several cross-sectional and meta-

Table 1. Mean values of ONSD, SR, and SWE in IIH patients versus controls, and in patients with mild versus moderate/severe papilledema

\begin{tabular}{lcccccc}
\hline & Patient $(\mathrm{n}=34)$ & Control $(\mathrm{n}=16)$ & P-value & Mild papilledema $(\mathrm{n}=49)$ & Moderate-severe papilledema $(\mathrm{n}=19)$ & P-value \\
\hline ONSD $(\mathrm{mm})$ & $5.6 \pm 1.1$ & $4.1 \pm 0.5$ & 0.001 & $5.4 \pm 0.9$ & $6.4 \pm 0.9$ & 0.001 \\
SR & $0.7 \pm 0.1$ & $0.9 \pm 0.1$ & 0.001 & $0.8 \pm 0.2$ & $0.6 \pm 0.1$ & 0.001 \\
SWE $(\mathrm{kPa})$ & $30.1 \pm 16.7$ & $8.2 \pm 3.4$ & 0.001 & $23.9 \pm 13.9$ & $46.2 \pm 11.9$ & 0.001 \\
\hline
\end{tabular}

ONSD, optic nerve sheath diameter; SR, strain ratio; SWE, shear wave elastography; IIH, idiopathic intracranial hypertension.

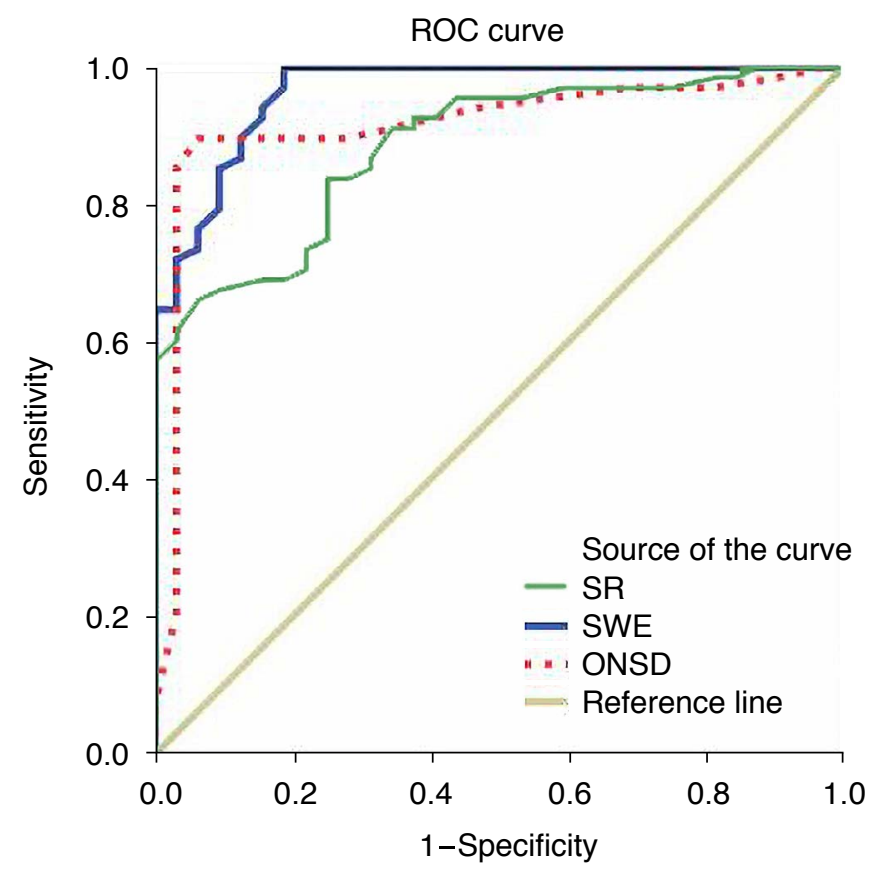

Diagonal segments are produced by ties

A

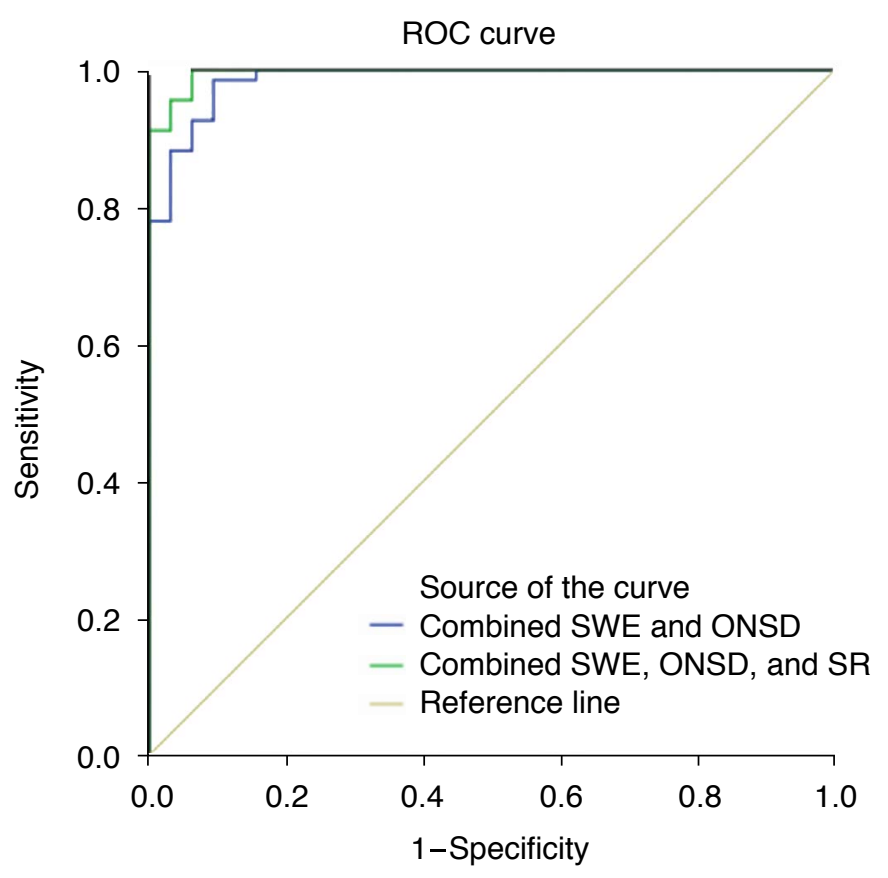

Fig. 3. Receiver operating characteristic (ROC) curves in idiopathic intracranial hypertension (IIH) patients versus the control group. ROC curves of strain ratio (SR), shear wave elastography (SWE), and optic nerve sheath diameter (ONSD) (A) and combined SWE and ONSD and combined SWE, ONSD, and SR (B) for differentiating IIH patients from control are shown. 
Table 2. The ROC curve results of ONSD, SR, SWE, and combined parameters in IIH patients versus the control group

\begin{tabular}{lcccccc}
\hline & Cutoff point & AUC & Sensitivity (\%) & Specificity (\%) & Accuracy (\%) & P-value \\
\hline ONSD & $5.45 \mathrm{~mm}$ & 0.91 & 89 & 93 & 91 & 0.001 \\
SR & 0.8 & 0.86 & 83 & 75 & 81 & 0.001 \\
SWE & $10.3 \mathrm{kPa}$ & 0.96 & 98 & 81 & 93 & 0.001 \\
Combined SWE and ONSD & & 0.98 & 98 & 90 & 96 & 0.001 \\
Combined SWE, ONSD, and SR & & 0.99 & 95 & 96 & 96 & 0.001 \\
\hline
\end{tabular}

ROC, receiver operating characteristic; ONSD, optic nerve sheath diameter; SR, strain ratio; SWE, shear wave elastography; IIH, idiopathic intracranial hypertension; AUC, area under the curve.

analysis studies have confirmed these results [18-20]. Generally, the reported normal and pathological ranges for ONSD varied from one study to the other; the variation in even pathological values in IIH patients varied as a result of the raised ICP due to other causes in previous investigations of neurocritical care patients [36-39]. The increased ONSD can be attributed to the fact that the ON is covered by a leptomeningeal sheath, which is expandable in the anterior segment, behind the globe. When ICP rises, the CSF is pushed towards the tiny rim of subarachnoid space between the sheath and the nerve, causing expansion of the dural covering. These changes are more marked in the anterior part of the nerve sheath behind the globe. As with any physiological change, the ONSD changes dynamically with changes in ICP [15-22].

A recent study evaluated the ON qualitatively using SE and quantitatively using SWE in IIH patients compared to a control group [29]. They found statistically significant differences in the types of elasticity strain patterns between both groups $(P=0.010)$. The uniqueness of our study is that it assessed the combination of ONSD with SR and SWE in the diagnosis of IIH.

We found that the SR was significantly lower in IIH patients than in healthy patients $(P=0.001)$, which indicates that with increased CSF pressure in the IIH, the CSF increased maximally around the anterior segment where the meningeal space was largest, and the stiffness of the anterior segment increased with a subsequent decrease in the SR. This may be explained by the fact that normally, the fenestrations of the lamina cribrosa and the surrounding glial columns form a tight seal around the intraocular segment to maintain a pressure gradient between the intraocular and intraorbital regions, allowing the axons to pass from an area of relatively higher intraocular pressure to an area of relatively lower CSF pressure. The meningeal ON sheath around the intraorbital segment is largest immediately behind the eyeball, narrower in the posterior part, and narrowest in the canalicular part. These facts may explain why the anterior segment of the intraorbital segment is more strongly affected by the increased CSF pressure [23-29]. To our knowledge, this is the first study to evaluate the SR of the ON.
No previous studies have assessed the SR of the $\mathrm{ON}$, either in $\mathrm{IH}$ or in any other condition.

In this study, the SWE indices were significantly higher in patients with $\mathrm{IH}(30.1 \pm 16.7 \mathrm{kPa})$ than in controls $(8.2 \pm 3.4 \mathrm{kPa})(\mathrm{P}=0.001)$, denoting the decreased elasticity of the $\mathrm{ON}$ in cases of increased ICP. Our results align with the most recent study that evaluated IIH patients' ON using SWE and reported significantly higher values of SWE in IIH patients $(26.9 \pm 1 \mathrm{kPa})$ than in the control group $(10.1 \pm 0.3$ $\mathrm{kPa}$ ) [29]. Previous studies reported increased ON stiffness in patients with multiple sclerosis $(33.9 \pm 11.6 \mathrm{kPa}$ for patients vs. $10.4 \pm 3.5 \mathrm{kPa}$ for controls), Behçet disease $(32.1 \pm 13.9 \mathrm{kPa}$ for patients vs. $11.5 \pm 5.6 \mathrm{kPa}$ for controls), and pre-eclampsia $(17.6 \pm 4.1$ $\mathrm{kPa}$ for patients vs. $9.4 \pm 2 \mathrm{kPa}$ for controls) including changes in the ON structure due to ischemic neuropathy, ON fibrosis, optic head swelling, and endothelial cell damage of the ON [23-27].

In the context of the pathophysiology of $\mathrm{IH}$, our results further support the role of ON swelling, as evidenced by the increased ONSD and papilledema, in addition to cellular damage, as evidenced by decreased $\mathrm{ON}$ elasticity.

As a multiparametric combination, increased ONSD values and decreased elasticity of the ON (as indicated by the SWE and/or SR) provide a better diagnostic tool for $\mathrm{IH}$ and a better indicator of the extent of papilledema. This may be attributed to the IIH-associated cascade of events: the increased amount of CSF leads to increased $I C P$, compressing the $\mathrm{ON}$ and resulting in venous congestion, papilledema, and cellular damage, which manifests clinically as blurring of vision and field defects. The combination of ONSD as a marker of papilledema and the SR and SWE as markers of cellular loss logically correlates with increased severity of the condition.

In this study, papilledema revealed a positive correlation with SWE and ONSD and a negative correlation with SR, with SWE being the most reliable parameter. Papilledema is a known phenomenon in conditions involving high ICP, and is related to the transmission of high ICP to the optic disc through the optic sheath that surrounds the subarachnoid space. Chronic venous engorgement also contributes to this high pressure [7]. 
There are some limitations of our study. First, the number of patients was relatively small, and the sample included only women. Further large-sample multicenter studies including both sexes are recommended. Second, this study used different ultrasound modalities (B-mode, strain, and shear elastography) to evaluate the ON in IIH patients. Third, ultrasonography (including elastography) is an operator-dependent technique; however, we tried to minimize this limitation by calculating the SR from three frames, and the SWE from three measurements and the mean values of these measurements were used for subsequent statistical analyses. Fourth, the orbit is an anatomical structure where very limited elastography studies have been performed. Further studies of orbital elastography incorporating interobserver variability are warranted. The combination of these ultrasound modalities with different multiparametric MR imaging modalities of the $\mathrm{ON}$ such as diffusion tensor imaging may improve results in the future.

We concluded that the combination of ONSD, SR, and SWE can help in the diagnosis of $\mathrm{IH}$ and serve as a good indicator of the extent of papilledema, with SWE being the most reliable parameter.

ORCID: Ahmed Abdel Khalek Abdel Razek: https://orcid.org/0000-0002-9613-5932; Nada Elsaid: https://orcid.org/0000-0002-2476-5652; Tamer Belal: https://orcid.org/00000002-3470-0668; Nihal Batouty: https://orcid.org/0000-0002-4544-2692; Ahmed Azab: https://orcid.org/0000-0002-6314-4824

\section{Author Contributions}

Conceptualization: Razek AAKA, Elsaid N, Belal T. Data acquisition: Razek AAKA, Elsaid N, Belal T, Batouty N, Azab A. Data analysis or interpretation: Razek AAKA, Batouty N. Drafting of the manuscript: Razek AAKA, Belal T, Batouty N, Azab A. Critical revision of the manuscript: Razek AAKA, Elsaid N, Azab A. Approval of the final version of the manuscript: all authors.

\section{Conflict of Interest}

No potential conflict of interest relevant to this article was reported.

\section{References}

1. Fargen KM. Idiopathic intracranial hypertension is not idiopathic: proposal for a new nomenclature and patient classification. J Neurointerv Surg 2020;12:110-114.

2. Ahmad SR, Moss HE. Update on the diagnosis and treatment of idiopathic intracranial hypertension. Semin Neurol 2019;39:682691.

3. Bouffard MA. Fulminant idiopathic intracranial hypertension. Curr Neurol Neurosci Rep 2020;20:8.

4. Radojicic A, Vukovic-Cvetkovic V, Pekmezovic T, Trajkovic G, Zidverc-
Trajkovic J, Jensen RH. Predictive role of presenting symptoms and clinical findings in idiopathic intracranial hypertension. J Neurol Sci 2019;399:89-93.

5. Madriz Peralta G, Cestari DM. An update of idiopathic intracranial hypertension. Curr Opin Ophthalmol 2018;29:495-502.

6. Wall M. Update on idiopathic intracranial hypertension. Neurol Clin 2017;35:45-57.

7. Elsaid N, Ahmed O, Belal T, Razek A, Azab A. Pathogenesis and evaluation of the effects of idiopathic intracranial hypertension on the optic nerves. Neuroophthalmology 2020;44:281-289.

8. Wong H, Sanghera K, Neufeld A, Maxner C, Shankar JJ. Clinicoradiological correlation of magnetic resonance imaging findings in patients with idiopathic intracranial hypertension. Neuroradiology 2020;62:49-53.

9. Bidot $\mathrm{S}$, Saindane AM, Peragallo JH, Bruce BB, Newman NJ, Biousse V. Brain imaging in idiopathic intracranial hypertension. J Neuroophthalmol 2015;35:400-411.

10. Razek A, Batouty NM, Azab AG. Role of susceptibility-weighted imaging in patients with idiopathic intracranial hypertension. Jpn J Radiol 2020;38:740-745.

11. Bateman GA, Bateman AR. Differences in the calculated transvenous pressure drop between chronic hydrocephalus and idiopathic intracranial hypertension. AJNR Am J Neuroradiol 2019;40:68-73.

12. Golden E, Krivochenitser R, Mathews N, Longhurst C, Chen Y, Yu $J$, et al. Contrast-enhanced 3D-FLAIR imaging of the optic nerve and optic nerve head: novel neuroimaging findings of idiopathic intracranial hypertension. AJNR Am J Neuroradiol 2019;40:334339.

13. Micieli JA, Bruce BB, Vasseneix C, Blanch RJ, Berezovsky DE, Peragallo $\mathrm{JH}$, et al. Optic nerve appearance as a predictor of visual outcome in patients with idiopathic intracranial hypertension. $\mathrm{Br} J$ Ophthalmol 2019;103:1429-1435.

14. Razek AA, Batouty N, Fathy W, Bassiouny R. Diffusion tensor imaging of the optic disc in idiopathic intracranial hypertension. Neuroradiology 2018;60:1159-1166.

15. De Bernardo M, Vitiello L, Rosa N. Sonographic evaluation of optic nerve sheath diameter in idiopathic intracranial hypertension. J Clin Neurosci 2020;73:331-332.

16. Naldi A, Provero P, Vercelli A, Bergui M, Mazzeo AT, Cantello R, et al. Optic nerve sheath diameter asymmetry in healthy subjects and patients with intracranial hypertension. Neurol Sci 2020;41:329333.

17. Tekin Orgun L, Atalay HT, Arhan E, Aydin K, Serdaroglu A. Optic nerve ultrasonography in monitoring treatment efficacy in pediatric idiopathic intracranial hypertension. Childs Nerv Syst 2020;36:1425-1433.

18. Jeub M, Schlapakow E, Ratz M, Kindler C, Schievelkamp AH, Wabbels $B$, et al. Sonographic assessment of the optic nerve and 
the central retinal artery in idiopathic intracranial hypertension. J Clin Neurosci 2020;72:292-297.

19. Lochner P, Fassbender K, Knodel S, Andrejewski A, Lesmeister M, Wagenpfeil $G$, et al. B-mode transorbital ultrasonography for the diagnosis of idiopathic intracranial hypertension: a systematic review and meta-analysis. Ultraschall Med 2019;40:247-252.

20. Lochner P, Fassbender K, Lesmeister M, Nardone R, Orioli A, Brigo $F$, et al. Ocular ultrasound for monitoring pseudotumor cerebri syndrome. J Neurol 2018;265:356-361.

21. del Saz-Saucedo P, Redondo-Gonzalez O, Mateu-Mateu A, HuertasArroyo R, Garcia-Ruiz R, Botia-Paniagua E. Sonographic assessment of the optic nerve sheath diameter in the diagnosis of idiopathic intracranial hypertension. J Neurol Sci 2016;361:122-127.

22. Lochner P, Brio F, Zedde ML, Sanguigni S, Coppo L, Nardone R, et al. Feasibility and usefulness of ultrasonography in idiopathic intracranial hypertension or secondary intracranial hypertension. BMC Neurol 2016;16:85.

23. Zemanova M. Shear wave elastography in ophthalmic diagnosis. J Fr Ophtalmol 2019;42:73-80.

24. Asal N, Sayan CD, Gokcinar NB, Sahan MH, Dogan A, Inal M. Evaluation of the optic nerve using strain and shear-wave elastography in pre-eclampsia. Clin Radiol 2019;74:813.

25. Inal M, Tan S, Yumusak EM, Sahan MH, Alpua M, Ornek K. Evaluation of the optic nerve using strain and shear wave elastography in patients with multiple sclerosis and healthy subjects. Med Ultrason 2017;19:39-44.

26. Sahan MH, Dogan A, Inal M, Alpua M, Asal N. Evaluation of the optic nerve by strain and shear wave elastography in patients with migraine. J Ultrasound Med 2019;38:1153-1161.

27. Guazzaroni M, Ferrari D, Lamacchia F, Salimei F, Marsico S, Citraro $D$, et al. Biomechanical properties of optic nerve and retrobulbar structures with 2D and 3D shear wave elastography in patients affected by glaucoma. Clin Imaging 2020;61:106-114.

28. Ozdemir A, Sahan MH, Asal N, Inal M, Gungunes A. Evaluation of the medial rectus muscle and optic nerve using strain and shear wave elastography in Graves' patients. Jpn J Radiol 2020;38:10281035.
29. Asal N, Inal M, Sahan MH, Say B. Assessment of the optic nerve using strain and shear-wave elastography in patients with pseudotumour cerebri. Clin Radiol 2020;75:629-635.

30. Friedman DI, Liu GT, Digre KB. Revised diagnostic criteria for the pseudotumor cerebri syndrome in adults and children. Neurology 2013;81:1159-1165.

31. Scott CJ, Kardon RH, Lee AG, Frisen L, Wall M. Diagnosis and grading of papilledema in patients with raised intracranial pressure using optical coherence tomography vs clinical expert assessment using a clinical staging scale. Arch Ophthalmol 2010;128:705-711.

32. Wall M. The morphology of visual field damage in idiopathic intracranial hypertension: an anatomic region analysis. In: Mills RP, Heijl A, eds. Perimetry update. Amsterdam: Kugler Publications, 1991;20-7.

33. Wall M. Idiopathic intracranial hypertension. Neurol Clin 2010;28:593-617.

34. Wall M, White WN 2nd. Asymmetric papilledema in idiopathic intracranial hypertension: prospective interocular comparison of sensory visual function. Invest Ophthalmol Vis Sci 1998;39:134142.

35. Mollan SP, Davies B, Silver NC, Shaw S, Mallucci CL, Wakerley BR, et al. Idiopathic intracranial hypertension: consensus guidelines on management. J Neurol Neurosurg Psychiatry 2018;89:1088-1100.

36. Geeraerts T, Merceron S, Benhamou D, Vigue B, Duranteau J. Noninvasive assessment of intracranial pressure using ocular sonography in neurocritical care patients. Crit Care 2008;12:P117.

37. Dubourg J, Javouhey E, Geeraerts T, Messerer M, Kassai B. Ultrasonography of optic nerve sheath diameter for detection of raised intracranial pressure: a systematic review and meta-analysis. Intensive Care Med 2011;37:1059-1068.

38. Ertl M, Barinka F, Torka E, Altmann M, Pfister $K$, Helbig $H$, et al. Ocular color-coded sonography: a promising tool for neurologists and intensive care physicians. Ultraschall Med 2014;35:422-431.

39. Wang L, Feng L, Yao Y, Wang Y, Chen Y, Feng J, et al. Optimal optic nerve sheath diameter threshold for the identification of elevated opening pressure on lumbar puncture in a Chinese population. PLoS One 2015;10:e0117939. 